\title{
Electromagnetic Casimir densities for a cylindrical boundary in de Sitter spacetime
}

\section{F. Manukyan*}

Department of Physics and Mathematics, Gyumri State Pedagogical Institute, 3126 Gyumri, Armenia

E-mail: mvardan_1972@mail.ru

\section{A. A. Saharian}

Department of Physics, Yerevan State University, 1 Alex Manoogian Street, 0025 Yerevan, Armenia

E-mail: saharianeysu.am

\begin{abstract}
Quantum vacuum effects are investigated for the electromagnetic field, induced by a conducting cylindrical shell in background of $(D+1)$-dimensional de Sitter spacetime. We assume that the field is prepared in the Bunch-Davies vacuum state. A complete set of the electromagnetic field mode functions is determined. By using these mode functions, the vacuum expectation value (VEV) of the electric field squared is evaluated for both exterior and interior regions. The VEV is decomposed into the boundary-free and boundary-induced contributions. The behavior of the latter is investigated in various asymptotic regions of the parameters. For $D=3$ the boundaryinduced VEV is related to the corresponding result for a cylindrical shell in Minkowski spacetime by standard conformal relation.
\end{abstract}

The Modern Physics of Compact Stars 2015 30 September 2015 - 3 October 2015

Yerevan, Armenia

${ }^{*}$ Speaker. 


\section{Introduction}

The de Sitter (dS) spacetime is the maximally symmetric solution of the Einstein equations with a positive cosmological constant. Its importance in gravitational physics is motivated by several reasons. First, because of the high symmetry, a large number of physical problems are exactly solvable on its background. A better understanding of the influence of the gravitational field on physical processes on the dS bulk could serve as a way to deal with more complicated geometries. The investigation of physical effects in dS spacetime is important for understanding the Universe at both the early and late stages of its expansion. In most inflationary scenarios [1], the dS spacetime is employed to solve a number of problems in standard cosmology. During an inflationary epoch, the quantum fluctuations generate seeds for the formation of large scale structures in the Universe. More recently, cosmological observations have indicated that the expansion of the Universe at the present epoch is accelerating [2] and the corresponding dynamics are well described by the model, with a positive cosmological constant as a dominant source. For this source, the standard cosmology would lead to an asymptotic dS universe in the future.

In dS spacetime, the interaction of fluctuating quantum fields with the background gravitational field gives rise to vacuum polarization. Another kind of vacuum polarization is induced by the presence of boundaries. This effect, known as the Casimir effect (for reviews see [3]), is among the most interesting macroscopic manifestations of nontrivial properties of the quantum vacuum. The imposition of boundary conditions on quantum fields alters the spectrum of the zero-point fluctuations and shifts the vacuum expectation values (VEVs) of physical quantities, such as the energy density and stresses. As a consequence, forces arise acting on constraining boundaries. The particular features of these forces depend on the nature of the quantum field, the type of spacetime manifold, the boundary geometry, and the specific boundary conditions imposed on the field.

In the present paper, for the electromagnetic field, we consider a problem with both types of vacuum polarization coming from the background geometry and boundaries. Namely, we evaluate the vacuum expectation value for the electric field squared induced by a cylindrical shell on the background of $(D+1)$-dimensional dS spacetime. The study of the Casimir effect for geometries involving cylindrical boundaries have attracted considerable theoretical and experimental interest. In most studies (see, for example, references given in [4]) the geometry of the background spacetime is Minkowskian. Combined effects of a cylindrical boundary and nontrivial topology induced by a cosmic string are discussed in [5]. In [6, 7, 4] the Casimir densities for a scalar field are investigated for planar, spherically and cylindrically symmetric boundaries in dS spacetime.

The paper is organized as follows. In the next section we present the cylindrical modes for the electromagnetic field in $\mathrm{dS}$ spacetime with an arbitrary number of spatial dimensions in the presence of a cylindrical shell on which the field obeys perfect conductor boundary conditions. By using these modes, in sections 3 and 4, we evaluate the VEV of the electric field squared inside and outside the cylindrical shell. This VEV is among the most important characteristics of the electromagnetic vacuum. The main results are summarized in section 5.

\section{Electromagnetic cylindrical modes in dS spacetime}

We consider a quantum electromagnetic field in background of a $(D+1)$-dimensional dS 
spacetime, in the presence of a perfectly conducting cylindrical shell having the radius $a$. In accordance with the problem symmetry, we will write the corresponding line element in cylindrical coordinates $(r, \phi, \mathbf{z})$ :

$$
d s^{2}=d t^{2}-e^{2 t / \alpha}\left[d r^{2}+r^{2} d \phi^{2}+(d \mathbf{z})^{2}\right],
$$

where $\mathbf{z}=\left(z^{3}, \ldots, z^{D}\right)$. The parameter $\alpha$ is related to the cosmological constant $\Lambda$ through the expression $\alpha^{2}=D(D-1) /(2 \Lambda)$. In addition to the synchronous time coordinate $t$, it is convenient to introduce the conformal time $\tau$ in accordance with $\tau=-\alpha e^{-t / \alpha},-\infty<\tau<0$. In terms of this coordinate the metric tensor takes a conformally flat form: $g_{i k}=(\alpha / \tau)^{2} \eta_{i k}$, with $\eta_{i k}$ being the Minkowskian metric tensor in Cartesian coordinates.

Here we are interested in the changes of the VEVs for the electromagnetic field induced by the cylindrical boundary. For a free electromagnetic field the Maxwell equations have the form

$$
\frac{1}{\sqrt{|g|}} \partial_{v}\left(\sqrt{|g|} F^{\mu v}\right)=0
$$

where $g$ is the determinant of the metric tensor and $F_{\mu \nu}$ is the electromagnetic field tensor, $F_{\mu \nu}=$ $\partial_{\mu} A_{v}-\partial_{v} A_{\mu}$. We assume that on the surface $r=a$ the field obeys the boundary condition

$$
n^{\mu} F_{\mu v_{1} \cdots v_{D-1}}^{*}=0,
$$

where $n^{\mu}$ is the normal to the boundary, $F_{\mu v_{1} \cdots v_{D-1}}^{*}$ is the dual of the field tensor $F_{\mu \nu}$. For $D=3$ the condition (2.3) reduces to the boundary condition on the surface of a perfect conductor. The VEV for a physical quantity $F\left\{A_{\mu}, A_{v}\right\}$, bilinear in the field operator, can be evaluated by using the mode-sum formula

$$
\left\langle 0\left|F\left\{A_{\mu}, A_{v}\right\}\right| 0\right\rangle=\sum_{\beta} F\left\{A_{(\beta) \mu}, A_{(\beta) v}^{*}\right\},
$$

where $\left\{A_{(\beta) \mu}, A_{(\beta) v}^{*}\right\}$ is a complete set of solutions of the classical field equations obeying the boundary conditions of the problem. Here $\beta$ stands for a set of quantum numbers specifying the modes. Hence, for the evaluation of the VEVs, in this scheme one needs to know the corresponding mode functions.

In what follows we will work in the coordinates $(\tau, r, \phi, \mathbf{z})$. In the Coulomb gauge one has $A^{0}=0, \partial_{l}\left(\sqrt{|g|} A^{l}\right)=0, l=1, \ldots, D$. For the geometry under consideration, the latter equation is reduced to $\partial_{l}\left(r A^{l}\right)=0$ which is the same as that in the Minkowski spacetime. If we present the solution in the factorized form, $A_{(\beta) i}=T(\tau) S_{(\beta) i}\left(x^{l}\right)$, then it can be shown that the parts of the mode functions corresponding to $S_{(\beta) i}\left(x^{l}\right)$ can be found in a way similar to the for the Minkowski bulk, whereas for the function $T(\tau)$ one gets $T(\tau)=\eta^{D / 2-1} Z_{D / 2-1}(\omega \eta)$ (for the definition of $\omega$ see below), where $\eta=|\tau|$ and $Z_{v}(x)$ is a cylinder function of the order $v$. It can be taken as a linear combination of the Hankel functions $H_{v}^{(1,2)}(x)$. The relative coefficient in the linear combination depends on the vacuum state under consideration. We assume that the field is prepared in the Bunch-Davies vacuum for which $Z_{v}(x)=H_{v}^{(1)}(x)$.

As a result, for the modes realizing the Bunch-Davies vacuum state in the region inside the cylindrical shell, $r<a$, one has the expressions

$$
\mathbf{A}_{(\beta)}=C_{\beta} \eta^{D / 2-1} H_{D / 2-1}^{(1)}(\omega \eta)\left(\frac{i m}{r},-r \partial_{r}, 0, \ldots, 0\right) J_{m}(\gamma r) e^{i m \phi+i \mathbf{k} \cdot \mathbf{z}},
$$


for the polarization $\sigma=1$, and

$$
\mathbf{A}_{(\beta)}=\omega C_{\beta} \eta^{D / 2-1} H_{D / 2-1}^{(1)}(\omega \eta)\left(\varepsilon_{\sigma}-\frac{i}{\omega^{2}} \sum_{l=3}^{D} k_{l} \varepsilon_{\sigma}^{l} \nabla\right) J_{m}(\gamma r) e^{i m \phi+i \mathbf{k} \cdot \mathbf{z}}
$$

for the polarizations $\sigma=2, \ldots, D-1$. Here, $\mathbf{A}_{(\beta)}=\left(A_{(\beta) 1}, \ldots, A_{(\beta) D}\right), m=0, \pm 1, \pm 2, \ldots, J_{m}(x)$ is the Bessel function, $\mathbf{k}=\left(k_{3}, \ldots, k_{D}\right), k=|\mathbf{k}|, \nabla=\left(\partial_{r}, \partial_{\phi}, \partial_{z_{1}}, \ldots, \partial_{z_{D}}\right)$, and

$$
\omega^{2}=\gamma^{2}+k^{2}
$$

For the polarization vectors $\varepsilon_{\sigma}=\left(0,0, \varepsilon_{\sigma}^{3}, \ldots, \varepsilon_{\sigma}^{D}\right), \sigma=2, \ldots, D-1$, one has the relations

$$
\sum_{l, n=3}^{D}\left(\omega^{2} \delta_{n}^{l}+k_{n} k^{l}\right) \varepsilon_{\sigma l} \varepsilon_{\sigma^{\prime}}^{n}=-\gamma^{2} \delta_{\sigma \sigma^{\prime}}
$$

and

$$
\sum_{\sigma=2}^{D-1} \varepsilon_{\sigma \mu} \varepsilon_{\sigma}^{v}=\left(k_{\mu} k^{v}-\gamma^{2} \delta_{\mu}^{v}\right) / \omega^{2}
$$

The eigenvalues for the quantum number $\gamma$ are determined by the boundary condition (2.3). For the mode $\sigma=1$ the allowed values for $\gamma$ are roots of the equation $J_{m}^{\prime}(\gamma a)=0$. For the modes $\sigma=2, \ldots, D-1$, the radial derivative enters in the expression for the component $A_{1}$ only. For these modes the boundary condition is reduced to $J_{m}(\gamma a)=0$. In what follows we will denote the eigenmodes by $\gamma a=\gamma_{m, n}^{(\lambda)}, n=1,2, \ldots$, where $\lambda=1$ for $\sigma=1$ and $\lambda=0$ for $\sigma=2,3, \ldots, D-1$ and, hence,

$$
J_{m}^{(\lambda)}\left(\gamma_{m, n}^{(\lambda)}\right)=0
$$

where $f^{(0)}(x)=f(x)$ and $f^{(1)}(x)=f^{\prime}(x)$. Consequently, in the interior region for the set of quantum numbers specifying the modes one has $\beta=(n, m, \sigma, \mathbf{k})$.

The normalization coefficients $C_{\beta}$ in (2.5) and (2.6) are determined from the orthonormalization condition for the vector potential:

$$
\int d^{D} x \sqrt{|g|}\left[A_{\left(\beta^{\prime}\right) v}^{*}(x) \nabla^{0} A_{(\beta)}^{v}(x)-\nabla^{0} A_{\left(\beta^{\prime}\right) v}^{*}(x) A_{(\beta)}^{v}(x)\right]=4 i \pi \delta_{\beta \beta^{\prime}}
$$

where the integration over the radial coordinate goes over the region inside the cylinder. By using the relation (2.8) it can be seen that the modes (2.6) are orthogonal. From (2.11) one finds

$$
\left|C_{\sigma}\right|^{2}=\frac{T_{m}(\gamma a)}{2(2 \pi \alpha)^{D-3} a \gamma}
$$

for $\sigma=1, \ldots, D-1$, where we have introduced the notation

$$
T_{m}(x)=x\left[x^{2} J_{m}^{\prime 2}(x)+\left(x^{2}-m^{2}\right) J_{m}^{2}(x)\right]^{-1} .
$$




\section{VEV of the electric field squared inside the shell}

As a local characteristic of the vacuum state we consider the VEV of the electric field squared. This VEV is obtained by making use of the mode-sum formula

$$
\left\langle 0\left|E^{2}(x)\right| 0\right\rangle \equiv\left\langle E^{2}(x)\right\rangle=-g^{00} g^{i k} \sum_{\beta} \partial_{0} A_{\beta i}(x) \partial_{0} A_{\beta k}^{*}(x) .
$$

The expression in the right-hand side is divergent and requires a regularization with the subsequent renormalization. The regularization can be done by introducing a cutoff function or by the point splitting. The consideration below does not depend on the specific regularization scheme. Substituting the eigenfunctions (2.5) and (2.6) into the mode-sum, for the VEV inside the shell we find

$$
\begin{aligned}
\left\langle E^{2}\right\rangle= & \frac{2^{6} A_{D} \eta^{D+2}}{\alpha^{D+1} a^{4}} \sum_{m=0}^{\infty} \int_{0}^{\infty} d k k^{D-3} \sum_{\lambda=0,1} \sum_{n=1}^{\infty} T_{m}\left(\gamma_{m, n}^{(\lambda)}\right) \\
& \times \gamma_{m, n}^{(\lambda) 3} F_{m}^{(\lambda)}\left[k, J_{m}\left(\gamma_{m, n}^{(\lambda)} r / a\right)\right] L_{D / 2-2}(\omega \eta),
\end{aligned}
$$

where the prime on the sign of the sum means that the term $m=0$ should be taken with the coefficient $1 / 2$,

$$
A_{D}=\frac{1}{(4 \pi)^{D / 2} \Gamma(D / 2-1)}
$$

and we have used the notation

$$
L_{v}(x)=K_{v}\left(x e^{-i \pi / 2}\right) K_{v}\left(x e^{i \pi / 2}\right),
$$

Here, instead of the Hankel functions we have introduced the Macdonald function $K_{v}(x)$. The function $F_{m}^{(\lambda)}[k, f(x)]$ is defined by the relations

$$
F_{m}^{(\lambda)}[k, f(x)]=\left\{\begin{array}{cc}
\left(k^{2} r^{2} / x^{2}\right)\left[f^{\prime 2}(x)+m^{2} f^{2}(x) / x^{2}\right]+\left[(D-3)\left(1+k^{2} r^{2} / x^{2}\right)+1\right] f^{2}(x), & \lambda=0, \\
\left(1+k^{2} r^{2} / x^{2}\right)\left[f^{\prime 2}(x)+m^{2} f^{2}(x) / x^{2}\right], & \lambda=1 .
\end{array}\right.
$$

The eigenvalues $\gamma_{m, n}^{(\lambda)}$ are given implicitly, as roots of the equation (2.10), and the representation (3.2) is not convenient for the further investigation of the VEV. In order to evaluate the mode-sum in (3.2), we apply to the series over $n$ the generalized Abel-Plana summation formula [8]

$$
\begin{array}{r}
\sum_{n=1}^{\infty} T_{m}\left(\gamma_{m, n}^{(\lambda)}\right) f\left(\gamma_{m, n}^{(\lambda)}\right)=\frac{1}{2} \int_{0}^{\infty} d x f(x)+\frac{\pi}{4} \operatorname{Res}_{z=0} f(z) \frac{Y_{m}^{(\lambda)}(z)}{J_{m}^{(\lambda)}(z)} \\
-\frac{1}{2 \pi} \int_{0}^{\infty} d x \frac{K_{m}^{(\lambda)}(x)}{I_{m}^{(\lambda)}(x)}\left[e^{-m \pi i} f\left(x e^{i \pi / 2}\right)+e^{m \pi i} f\left(x e^{-i \pi / 2}\right)\right],
\end{array}
$$

where $Y_{m}(z)$ is the Neumann function and $I_{m}(x)$ is the modified Bessel function of the first kind. In (3.6) it is assumed that the function $f(z)$ is analytic in the right half plane of the complex variable $z$. For the series in (3.2), as the function $f(z)$ we take

$$
f(z)=z^{3} L_{D / 2-2}\left(\eta \sqrt{z^{2} / a^{2}+k^{2}}\right) F_{m}^{(\lambda)}\left[k, J_{m}(z r / a)\right] .
$$


After the application of the summation formula, the VEV of the electric field squared is decomposed as

$$
\left\langle E^{2}\right\rangle=\left\langle E^{2}\right\rangle_{0}+\left\langle E^{2}\right\rangle_{\mathrm{b}}
$$

Here, the first term in the right-hand side comes from the first integral in (3.6) and is the VEV in dS spacetime in the absence of boundaries:

$$
\left\langle E^{2}\right\rangle_{0}=\frac{2^{5} A_{D} \eta^{D+2}}{\alpha^{D+1}} \sum_{m=0}^{\prime} \int_{0}^{\infty} d k k^{D-3} \int_{0}^{\infty} d x x^{3} F_{(0) m}\left[k, J_{m}(x r)\right] L_{D / 2-2}\left(\eta \sqrt{k^{2}+x^{2}}\right),
$$

where

$$
\begin{aligned}
F_{(0), m}[k, f(x)]= & \left(1+2 \frac{k^{2} r^{2}}{x^{2}}\right)\left[f^{\prime 2}(x)+\frac{m^{2}}{x^{2}} f^{2}(x)\right] \\
& +\left[(D-3)\left(1+\frac{k^{2} r^{2}}{x^{2}}\right)+1\right] f^{2}(x) .
\end{aligned}
$$

The part of the VEV $\left\langle E^{2}\right\rangle_{\mathrm{b}}$ is the contribution of the last integral of (3.6). This part is induced by the presence of the cylindrical boundary and is given by the expression

$$
\begin{aligned}
\left\langle E^{2}\right\rangle_{\mathrm{b}}= & \frac{2^{5} A_{D}}{\alpha^{D+1}} \sum_{m=0}^{\prime} \sum_{\lambda=0,1} \int_{0}^{\infty} d x x^{D+1} \frac{K_{m}^{(\lambda)}(x a / \eta)}{I_{m}^{(\lambda)}(x a / \eta)} \\
& \times \int_{0}^{1} d s s\left(1-s^{2}\right)^{D / 2-2} G_{m}^{(\lambda)}\left[s, I_{m}(x r / \eta)\right] f_{D / 2-2}(x s),
\end{aligned}
$$

with the notations

$$
f_{v}(x)=K_{v}(x)\left[I_{-v}(x)+I_{v}(x)\right]
$$

and

$$
G_{m}^{(\lambda)}[s, f(x)]=\left\{\begin{array}{cl}
\left(1-s^{2}\right)\left[f^{\prime 2}(x)+m^{2} f^{2}(x) / x^{2}\right]+\left[s^{2}(D-3)+1\right] f^{2}(x), \lambda=0, \\
-s^{2}\left[f^{\prime 2}(x)+m^{2} f^{2}(x) / x^{2}\right], & \lambda=1 .
\end{array}\right.
$$

The representation (3.11) is valid for all even values of $D$ and for $D<7$ in the case of odd $D$.

As is seen from (3.9) and (3.11), in the new representation of the VEV the explicit knowledge of the eigenvalues $\gamma_{m, n}^{(\lambda)}$ is not required. Another advantage is that we have explicitly separated the part corresponding to the boundary-free dS spacetime. The presence of the boundary does not change the local geometry for points outside of the shell. This means that at those points the divergences are the same in both problems, in the absence and in the presence of the boundary. From here it follows that the divergences in (3.8) are contained in the part $\left\langle E^{2}\right\rangle_{0}$ only, whereas the boundary-induced contribution $\left\langle E^{2}\right\rangle_{\mathrm{b}}$ is finite for points away from the boundary and the regularization, implicitly assumed in the discussion above, can be safely removed. Hence, the renormalization is required for the boundary-free part only. Note that the expression for the latter can be further simplified after the summation over $m$ by using the standard result for the series involving the square of the Bessel function.

Let us consider the properties of the boundary-induced contribution in the VEV of the field squared. First of all, by taking into account that for $D \geqslant 3$ one has $G_{m}^{(0)}[s, f(x)]>0, G_{m}^{(1)}[s, f(x)]<$ 
0 , and the function $f_{D / 2-2}(x)$ is positive for the values of $D$ for which the representation (3.11) is valid, from (3.11) it follows that $\left\langle E^{2}\right\rangle_{\mathrm{b}}$ is always positive. The VEV of the electric field squared inside a cylindrical shell in Minkowski spacetime is obtained the limiting transition $\alpha \rightarrow \infty$ for a fixed value of $t$. In this limit one has $\eta \approx \alpha-t$ and, hence, $\eta$ is large. Passing to a new integration variable $y=x / \eta$, we see that $\eta$ appears in the argument of the function $f_{D / 2-2}(y s \eta)$. By taking into account that for large arguments one has $f_{D / 2-2}(u) \approx 1 / u$, after the integration over $s$, one gets $\lim _{\alpha \rightarrow \infty}\left\langle E^{2}\right\rangle_{b}=\left\langle E^{2}\right\rangle_{(\mathrm{M}) b}$, where

$$
\left\langle E^{2}\right\rangle_{(\mathrm{M}) b}=\frac{4(4 \pi)^{(1-D) / 2}}{\Gamma((D+1) / 2)} \sum_{m=0}^{\prime} \sum_{\lambda=0,1} \int_{0}^{\infty} d x x^{D} \frac{K_{m}^{(\lambda)}(a x)}{I_{m}^{(\lambda)}(a x)} G_{(\mathrm{M}) m}^{(\lambda)}\left[I_{m}(r x)\right]
$$

with

$$
G_{(\mathrm{M}) m}^{(\lambda)}[f(x)]=\left\{\begin{array}{cc}
(D-2)\left[f^{\prime 2}(x)+\left(m^{2} / x^{2}+2\right) f^{2}(x)\right], & \lambda=0, \\
-\left[f^{\prime 2}(x)+m^{2} f^{2}(x) / x^{2}\right], & \lambda=1,
\end{array}\right.
$$

is the VEV of the electric field squared inside a cylindrical shell in the Minkowski bulk.

In the special case of 4-dimensional dS spacetime one has $D=3$ and $f_{-1 / 2}(u)=1 / u$. After the integration over $s$ in (3.11), we find

$$
\left\langle E^{2}\right\rangle_{b}=(\eta / \alpha)^{4}\left\langle E^{2}\right\rangle_{(\mathbf{M}) b}
$$

where $\left\langle E^{2}\right\rangle_{(\mathrm{M}) b}$ is given by (3.14) with $D=3$. In this case, the VEV of the field squared is related to the corresponding result in Minkowski spacetime by standard conformal transformation with the conformal factor $(\eta / \alpha)^{4}$. This is a direct consequence of the conformal invariance of the electromagnetic field in $D=3$ spatial dimensions and of the conformal flatness of the background geometry. Note that the Casimir energy of an infinite perfectly conducting cylindrical shell in background of 4-dimensional Minkowski spacetime has been evaluated in [9] on the base of a Green's function technique with an ultraviolet regulator. Later the corresponding result was rederived by the zeta function technique $[10,11]$ and by using the mode-by-mode summation technique [12]. The VEV of the energy-momentum tensor inside and outside of the shell has been investigated in [13].

On the axis of the shell, $r=0$, the only nonzero contribution to the boundary-induced VEV comes from the terms in (3.11) with $m=0,1$ :

$$
\begin{aligned}
\left\langle E^{2}\right\rangle_{\mathrm{b}, r=0}= & \frac{2^{4} A_{D}}{\alpha^{D+1}}\left(\frac{\eta}{a}\right)^{D+2} \int_{0}^{\infty} d x x^{D+1} \int_{0}^{1} d s s\left(1-s^{2}\right)^{D / 2-2} f_{D / 2-2}(x s \eta / a) \\
& \times\left[\left(s^{2}(D-3)+1\right) \frac{K_{0}(x)}{I_{0}(x)}+\left(1-s^{2}\right) \frac{K_{1}(x)}{I_{1}(x)}-s^{2} \frac{K_{1}^{\prime}(x)}{I_{1}^{\prime}(x)}\right] .
\end{aligned}
$$

The shell-induced VEV diverges on the boundary. For points near the boundary the contribution of the modes with small wavelengths dominate and at distances from the shell smaller than the curvature radius of the $\mathrm{dS}$ spacetime the influence of the gravitational field is small. The leading term in the expansion with respect to the distance coincides with that for the shell in Minkowski bulk. 


\section{Field squared in the exterior region}

In the region outside the shell, the eigenfunctions for the vector potential, obeying the boundary condition (2.3), are given by the formulae (2.5) and (2.6) with the replacement

$$
J_{m}(\gamma r) \rightarrow g_{m}^{(\lambda)}(\gamma a, \gamma r)=J_{m}(\gamma r) Y_{m}^{(\lambda)}(\gamma a)-Y_{m}(\gamma r) J_{m}^{(\lambda)}(\gamma a) .
$$

Now, in the normalization condition (2.11) the integration over the radial coordinate goes over the region $a \leqslant r<\infty$ and in the right-hand side the delta symbol is understood as the Dirac delta function $\delta\left(\gamma-\gamma^{\prime}\right)$. As the normalization integral diverges for $\gamma^{\prime}=\gamma$, the main contribution into the integral comes from large values of $r$. By making use of the asymptotic formulas for the Bessel and Neumann functions for large arguments, one gets

$$
\left|C_{\sigma}\right|^{2}=\frac{\left[J_{m}^{(\lambda) 2}(\gamma a)+Y_{m}^{(\lambda) 2}(\gamma a)\right]^{-1}}{4(2 \pi \alpha)^{D-3} \gamma} .
$$

For the VEV of the field squared one finds

$$
\begin{aligned}
\left\langle E^{2}\right\rangle= & \frac{2^{5} A_{D} \eta^{D+2}}{\alpha^{D+1}} \sum_{m=0}^{\infty} \int_{0}^{\infty} d k k^{D-3} \int_{0}^{\infty} d \gamma \\
& \times \gamma^{3} \sum_{\lambda=0,1} \frac{F_{m}^{(\lambda)}\left[k, g_{m}^{(\lambda)}(\gamma a, \gamma r)\right]}{J_{m}^{(\lambda) 2}(\gamma a)+Y_{m}^{(\lambda) 2}(\gamma a)} L_{D / 2-2}(\omega \eta) .
\end{aligned}
$$

Similar to the case of the interior region, we assume that some regularization scheme is used for the right-hand side of (4.3). In order to extract the boundary-free contribution, given by (3.9), we use the relation

$$
\frac{F_{m}^{(\lambda)}\left[k, g_{m}^{(\lambda)}(\gamma a, \gamma r)\right]}{J_{m}^{(\lambda) 2}(\gamma a)+Y_{m}^{(\lambda) 2}(\gamma a)}=F_{m}^{(\lambda)}\left[k, J_{m}(\gamma a)\right]-\frac{1}{2} \sum_{j=0,1} \frac{J_{m}^{(\lambda)}(\gamma a)}{H_{m}^{(j)(\lambda)}(\gamma a)} F_{m}^{(\lambda)}\left[k, H_{m}^{(j)}(\gamma a)\right] .
$$

This allows to present the VEV of the field squared in the decomposed form (3.8) with the shellinduced part

$$
\begin{aligned}
\left\langle E^{2}\right\rangle_{\mathrm{b}}= & -\frac{2^{4} A_{D} \eta^{D+2}}{\alpha^{D+1}} \sum_{m=0}^{\prime} \int_{0}^{\infty} d k k^{D-3} \sum_{j=0,1} \sum_{\lambda=0,1} \int_{0}^{\infty} d \gamma \\
& \times \gamma^{3} \frac{J_{m}^{(\lambda)}(\gamma a)}{H_{m}^{(j)(\lambda)}(\gamma a)} F_{m}^{(\lambda)}\left[k, H_{m}^{(j)}(\gamma a)\right] L_{D / 2-2}(\omega \eta) .
\end{aligned}
$$

Now, we rotate the contour of integration over $\gamma$ by the angle $\pi / 2$ for the term with $j=1$ and by the angle $-\pi / 2$ for $j=2$. Introducing the modified Bessel function, the expression (4.5) takes the form

$$
\begin{aligned}
\left\langle E^{2}\right\rangle_{\mathrm{b}}= & \frac{2^{5} A_{D}}{\alpha^{D+1}} \sum_{m=0}^{\prime} \sum_{\lambda=0,1} \int_{0}^{\infty} d x x^{D+1} \frac{I_{m}^{(\lambda)}(a x / \eta)}{K_{m}^{(\lambda)}(a x / \eta)} \\
& \times \int_{0}^{1} d s s\left(1-s^{2}\right)^{D / 2-2} G_{m}^{(\lambda)}\left[s, K_{m}(x r / \eta)\right] f_{D / 2-2}(x s),
\end{aligned}
$$


where the functions $G_{m}^{(\lambda)}[s, f(x)]$ are defined by the formulae (3.13). Comparing this result with (3.11), we see that the expressions for the interior and exterior regions are related by the interchange $I_{m} \leftrightarrows K_{m}$. The representation (4.5) is valid for even values of $D$ and for odd values $D<7$. Similar to the case of the interior region, for these values of $D$, the boundary-induced VEV is positive in the exterior region.

At large proper distances from the shell compared with the dS curvature radius, we have $r / \eta \gg 1$. Introducing in (4.5) a new integration variable $y=x r / \eta$, we expand the functions $I_{m}^{(\lambda)}(y a / r) / K_{m}^{(\lambda)}(y a / r)$ and $f_{D / 2-2}(y s \eta / r)$ for small values of the arguments. The leading contribution comes from the term with $\lambda=0, m=0$. For even $D>4$ one gets

$$
\left\langle E^{2}\right\rangle_{\mathrm{b}} \approx \frac{8(2 D+1)(D-2) \Gamma^{3}(D / 2+1)}{D(D-4) \Gamma(D+2) \pi^{D / 2} \alpha^{D+1} \ln (r / a)}(\eta / r)^{D+2} .
$$

In the case $D=4$ the leading term is given by

$$
\left\langle E^{2}\right\rangle_{\mathrm{b}} \approx \frac{12 \pi^{-2}(\eta / r)^{6}}{5 \alpha^{5} \ln (r / a) \ln (r / \eta)}
$$

For $D=3$ and $D=5$ we find

$$
\begin{aligned}
\left\langle E^{2}\right\rangle_{\mathrm{b}} & \approx \frac{2(\eta / r)^{4}}{3 \pi \alpha^{4} \ln (r / a)}, D=3, \\
\left\langle E^{2}\right\rangle_{\mathrm{b}} & \approx \frac{7(\eta / r)^{6}}{10 \pi^{2} \alpha^{6} \ln (r / a)}, D=5 .
\end{aligned}
$$

Note that for a cylindrical shell in Minkowski bulk, at large distance from the shell, $r \gg a$, the leading term in the corresponding asymptotic expansion is given by

$$
\left\langle E^{2}\right\rangle_{(\mathrm{M}) \mathrm{b}} \approx \frac{(3 D-1)(D-2) \Gamma^{3}((D+1) / 2)}{\pi^{(D-1) / 2}(D-1) \Gamma(D+1) r^{D+1} \ln (r / a)},
$$

for all dimensions with $D \geqslant 3$. As is seen, the influence of the gravitational field is essential at distances from the shell larger than the curvature radius of the background spacetime.

\section{Conclusion}

We have investigated the combined effects of the background gravitational field and boundaries on the characteristics of the electromagnetic vacuum. In order to have an exactly solvable problem we have considered highly symmetric bulk and boundary geometries: cylindrical shell in background of dS spacetime. On the shell the field operator obeys the boundary condition that is a generalization of the perfect conductor boundary condition for an arbitrary number of spatial dimensions. We have assumed that the field is prepared in the Bunch-Davies vacuum state. The VEVs of physical observables, bilinear in the field operator, are expressed in the form of the summation over a complete set of the field modes obeying the boundary conditions. We have constructed these modes for the problem under consideration. For separate polarizations, the corresponding vector potentials are given by the expressions (2.5) and (2.6) in the region inside the shell and the eigenvalues for $\gamma$ are determined by the equation (2.10). In the exterior region the eigenvalues of $\gamma$ are 
continuous and the mode functions are obtained from the interior ones by the replacement (4.1) in the radial functions.

As a physical characteristic of the vacuum state we have investigated the VEV of the electric field squared. In the corresponding mode-sum, for the summation over the eigenvalues of $\gamma$ we have used the summation formula (3.6). This allowed us to extract from the VEV the boundaryfree part and to present the contribution induced by the shell in the form of strongly convergent integral for points away from the boundary. In this way, the renormalization is reduced to the one for the boundary-free dS spacetime. The boundary-induced contributions to the VEV of the electric field squared in the interior and exterior regions are given by the expressions (3.11) and (4.6). In both the regions these contributions are positive. For points near the boundary, at distances from the shell smaller than the curvature radius of the dS spacetime, the influence of the gravitational field on the vacuum local characteristics is small. We have shown that the effect of the background curvature is essential at distances from the shell larger than the dS curvature radius.

\section{Acknowledgments}

A. A. S. was supported by the State Committee of Science Ministry of Education and Science RA, within the frame of Grant No. SCS 15T-1C110, and by the Armenian National Science and Education Fund (ANSEF) Grant No. hepth-4172.

\section{References}

[1] A. D. Linde, Particle Physics and Inflationary Cosmology (Harwood Academic Publishers, Chur, Switzerland 1990).

[2] A. G. Riess et al., Astrophys. J. 659, 98 (2007); D.N. Spergel et al., Astrophys. J. Suppl. Ser. 170, 377 (2007); E. Komatsu et al., Astrophys. J. Suppl. Ser. 180, 330 (2009).

[3] V. M. Mostepanenko, N. N. Trunov, The Casimir Effect and Its Applications (Oxford University Press, Oxford, 1997); K. A. Milton, The Casimir Effect: Physical Manifestation of Zero-Point Energy (World Scientific, Singapore, 2002); M. Bordag, G. L. Klimchitskaya, U. Mohideen, V. M. Mostepanenko, Advances in the Casimir Effect (Oxford University Press, Oxford, 2009); Casimir Physics, edited by D. Dalvit, P. Milonni, D. Roberts, F. da Rosa, Lecture Notes in Physics Vol. 834 (Springer-Verlag, Berlin, 2011).

[4] A. A. Saharian, V. F. Manukyan, Class. Quantum Grav. 32, 025009 (2015).

[5] I. Brevik, T. Toverud, Class. Quantum Gravity 12, 1229 (1995); E. R. Bezerra de Mello, V. B. Bezerra, A. A. Saharian, A. S. Tarloyan, Phys. Rev. D 74, 025017 (2006); E. R. Bezerra de Mello, V. B. Bezerra, A. A. Saharian, Phys. Lett. B 645, 245 (2007); E. R. Bezerra de Mello, V. B. Bezerra, A. A. Saharian, A. S. Tarloyan, Phys. Rev. D 78, 105007 (2008); V. V. Nesterenko, I. G. Pirozhenko, Class. Quantum Grav. 28, 175020 (2011).

[6] M. R. Setare, R. Mansouri, Classical Quantum Gravity 18, 2331 (2001); A. A. Saharian, T. A. Vardanyan, Class. Quantum Grav. 26, 195004 (2009); E. Elizalde, A. A. Saharian, T. A. Vardanyan, Phys. Rev. D 81, 124003 (2010); A. A. Saharian, Int. J. Mod. Phys. A 26, 3833 (2011); P. Burda, JETP Lett. 93, 632 (2011); S. Bellucci, A. A. Saharian, A. H. Yeranyan, Phys. Rev. D 89, 105006 (2014). 
[7] K. A. Milton, A. A. Saharian, Phys. Rev. D 85, 064005 (2012).

[8] A. A. Saharian, The Generalized Abel-Plana Formula with Applications to Bessel Functions and Casimir Effect (Yerevan State University Publishing House, Yerevan, 2008); Report No. ICTP/2007/082; arXiv:0708.1187.

[9] L. L. De Raad Jr., K. A. Milton, Ann. Phys. 136, 229 (1981).

[10] P. Gosdzinsky, A. Romeo, Phys. Lett. B 441, 265 (1998).

[11] G. Lambiase, V. V. Nesterenko, M. Bordag, J. Math. Phys. 40, 6254 (1999).

[12] K. A. Milton, A. V. Nesterenko, V. V. Nesterenko, Phys. Rev. D 59, 105009 (1999).

[13] A. A. Saharian, Izv. AN Arm. SSR. Fizika 23, 130 (1988) [Sov. J. Contemp. Phys. 23, 14 (1988)]. 